\title{
Consciousness, Quantum Physics, and Hermeneutical Phenomenology
}

Patrick Aidan Heelan S.J.

Georgetown University, heelanp@georgetown.edu

Follow this and additional works at: https://fordham.bepress.com/phil_research

Part of the Continental Philosophy Commons, Logic and Foundations of Mathematics Commons, Philosophy of Mind Commons, and the Philosophy of Science Commons

\section{Recommended Citation}

Heelan, Patrick Aidan S.J., "Consciousness, Quantum Physics, and Hermeneutical Phenomenology" (2014). Research Resources. 32. https://fordham.bepress.com/phil_research/32

This Article is brought to you for free and open access by the Hermeneutic and Phenomenological Philosophies of Science at DigitalResearch@Fordham. It has been accepted for inclusion in Research Resources by an authorized administrator of DigitalResearch@Fordham. For more information, please contact considine@fordham.edu. 


\title{
Consciousness, Quantum Physics, and Hermeneutical Phenomenology
}

\author{
Patrick Aidan Heelan
}

\begin{abstract}
Two hundred years ago Friedrich Schleiermacher (See Wellmon 2006) modified Kant's notion of anthropology—-hermeneutically,' as he said—so as to make it inclusive of the tribes that Captain Cook found in the South Sea Islands. This paper honors the late Joseph J. Kockelmans for making a similar hermeneutic move to update Kant's notion of natural science so as to make it inclusive of the phenomenological lifeworld (For 'lifeworld,' see Husserl's The Crisis of European Sciences and Transcendental Philosophy, 1954, 121-148, and the 'lifeworld' theme throughout the Crisis.) syntheses of classical, relativity, and quantum physics. The new synthesis is in fact not alien to the views of some of the founders of quantum mechanics, notably Eugene Wigner, John von Neumann, Paul Dirac, Werner Heisenberg - possibly even Albert Einstein. In this hermeneutical move, the 'observer' is 'embodied consciousness,' and 'measure-numbers' represent 'observable presence.' The new theoretical synthesis of physics is a representation
\end{abstract}

\footnotetext{
NOTES ON SOURCES: Some of the sources used in this paper are listed in the references below. Most of the referenced Heelan texts can be found on the website, https://gushare.georgetown.edu/ heelanp/ or http://fordham.bepress.com/phil_research/. In the field of mathematics and theoretical physics, I have learned from my physics mentors: from the lectures of Erwin Schrödinger and John Synge on classical non-Euclidean geometries, and from personal communications with and the publications of Nobelists Eugene Wigner (cf. 1963, 1967) and Werner Heisenberg (cf. 1950) on the role of subjectivity in assessing the rationality of the quantum theory. I have also profited from discussions on cognitive science with Karl Pribram and his writings (cf. 1971, 1991) on the building of a scientific model of human embodied consciousness. In linguistics, I have learnt much about language from my colleague in German Linguistics, Heidi Byrnes at Georgetown University. I owe a special debt to Babette Babich, at Fordham University, my former student, who has been a constant partner in scholarship for many years. These, among many others too numerous to mention, are the principal dialogical and dialectical sources of the rational heuristic I have used to explore the nature of the human consciousness and the Spirit that raises it up above pure Nature.
}

P.A. Heelan $(\bowtie)$

William Gaston Professor of Philosophy

Georgetown University, Georgetown, USA

e-mail: Babich@fordham.edu 
of a physical system as a dynamic Hilbert Vector Space; empirical 'observables' are represented by projection operators, each of which maps a subspace of definite measurable values. Among these projection operators, some pairs are 'complementary' and share a common subspace of the Hilbert Space where they can be precisely measured together in a common laboratory setting. Some pairs, however, are 'non-complementary' and do not share a common subspace; these lead to Uncertainty Principles of the quantum mechanical kind. The quantum notion of an "observable" introduces into the discursive language of physics the common sense lifeworld notion of "contextuality." This analysis completes Husserl's analysis of science in the Crisis, so well articulated and developed by Kockelmans (See Kockelmans' contributions to the phenomenology of natural science in Kockelmans and Kisiel (1970)).

\section{Phenomenology, Hermeneutics, and Quantum Theory}

It is only when we arrive at consciousness as the universal medium of access of whatever exists and has value, including the lifeworld itself, that our research for foundations reaches its final destination. In other words, our ontology of the lifeworld reaches its ultimate foundation only in the constitutive analyses of transcendental phenomenology.

— Joseph Kockelmans ${ }^{1}$

Physicists and philosophers of science are persuaded that epistemologically quantum mechanics departs radically from classical physics. Few, however, take seriously the insight of three of the founders of quantum physics-Erwin Schrödinger, Werner Heisenberg, and Eugene Wigner ${ }^{2}$ - that the strangeness of quantum physics involves the emergent epistemological and ontological role of embodied human consciousness in the process of measurement.

I am reminded of the following story told to me by Heisenberg in 1965. In April, 1926, before his paper on the Uncertainty Principles in Quantum Mechanics was published, Albert Einstein invited him to speak to the senior physicists in Berlin on this topic; Einstein presided at the meeting and, when Heisenberg had concluded his presentation, he spoke saying that all this uncertainty talk was nonsense. Much taken aback, Heisenberg responded that he was only applying the principle Einstein himself used in his 1905 relativity paper-that the measure-numbers of the mathematical theory described reality. To this, Einstein responded: "The

\footnotetext{
${ }^{1}$ Ibid, 67.

${ }^{2}$ I am a physicist who studied (1946-1948) relativistic cosmology with Erwin Schrödinger and John Synge at the Dublin Institute for Advanced Studies; later I studied as a post-doc (1960-1962) in high-energy quantum physics with Eugene Wigner at Princeton; and in 1962-1964, I visited frequently with Werner Heisenberg in Munich while writing a book on Heisenberg's philosophy of science (Heelan 1965). Out of my many discussions with them, I developed an interest in the way these three Nobel Prize physicists, interested in Husserl's philosophy, attributed a fundamental role to human consciousness in quantum physics.
} 
measure-number is to that which is measured as the number on your cloakroom ticket is to your overcoat-it tells you nothing about your overcoat." Einstein then invited Heisenberg to walk with him while they discussed the problem of quantum uncertainty. They walked and talked for an hour, and when they returned to the conference room, those who awaited their return asked what the outcome was. Einstein replied that he and Heisenberg now understood one another and were in agreement. When pressed to state what their agreement was, Einstein refused to speak about it. At that moment I asked Heisenberg what was their agreement. He replied that a distinction has to be made between the 'presence' of the 'real' and any 'intuition' that may have accompanied the sense of presence. I will return to this topic below. The aim of this paper is to reflect on and attempt to articulate the content of the agreement between Heisenberg and Einstein on that occasion in 1926.

In the tradition of classical physics, the observer is a disembodied mind external to nature, and the objective essence of nature is revealed in the mathematical intuition of its structure. In quantum mechanics, however, the observer is not a disembodied mind, nor does the mathematical intuition represent an 'objective' presence but the probability of a presence. ${ }^{3}$ In quantum mechanics, the observer is a human consciousness embodied in instruments that serve as a bodily extension of the observer's embodiment. The empirical observations of quantum physics consequently involve a bilateral relation between the observer's enhanced embodied consciousness (on the subject side) and what is observed (on the object side), each having its place and context within the lifeworld of Nature as culture. ${ }^{4}$ The concepts and judgments of quantum physics consequently are contextualized subjectively and objectively by the 'natural world' as structured by science.

Human consciousness makes meanings from its sensory engagement with nature through practices that are learned and later function at an unconscious anticipatory intentional level. ${ }^{5}$ For a deeper understanding of this process, I draw, not from Carnap's logical empiricism, but from the post-kantian German philosophies contemporary with the development of quantum mechanics. While quantum mechanics was taking shape in Göttingen, Leipzig, and Munich, where Heisenberg studied and worked, the phenomenology of Edmund Husserl was academically prominent. Later, Heisenberg was also one of a circle of professional scientific intellectuals who met regularly during the summer with Martin Heidegger in the Black Forest. ${ }^{6}$ In Germany, phenomenology and hermeneutics were wissenschaftlich approaches to psychology, art, literature, music, and natural science, establishing them both as 'scientific' and 'philosophical,' on a par academically with the role of logic and analysis in the contemporary USA. ${ }^{7}$ In addition to Husserl and Heidegger,

\footnotetext{
${ }^{3}$ See Kockelmans (1970a, c) in Kockelmans and Kisiel (1970).

${ }^{4}$ See ibid, Kockelmans (1970b).

${ }^{5}$ See ibid, Kockelmans and Kisiel (1970).

${ }^{6}$ Heisenberg contributed an essay on the Uncertainty Principle to a Festschrift to honor Heidegger on his 70th birthday.

${ }^{7}$ See Kisiel (1970a, b) in Kockelmans and Kisiel (1970).
} 
Dilthey's work on history, Maurice Merleau-Ponty's work on perception, ${ }^{8}$ and Hans-Georg Gadamer's work on literature and art also inspired a strong current in European academic culture before and after the war. These currents of thought also inspired Michael Polanyi, who was Wigner's scientific mentor. Looking back on his life as a physicist, Wigner told his chronicler, Andrew Szanton:

My chief scientific interest in the last twenty years has been to somehow extend theoretical physics into the realm of consciousness... Consciousness is beautifully complex. It has never been properly described, certainly not by physics and mathematics. It is shrouded in mysteries. And what I know of philosophy and psychology suggests that these disciplines have never defined consciousness either. (Szanton 1992, p. 309).

\section{Human Consciousness as the 'Governor of Mental Life'}

What is 'human consciousness'? Few cognitive scientists are willing to define it, perhaps, because a human subject trying to define it objectively leads to an infinite series of recurrent questions! Human consciousness certainly processes information signals - but so does Deep Blue, the IBM computer chess champion; but, in addition, it has sensory experiences, produces new insights, tests for relevant truth in the world, and makes free value-laden decisions on the basis of the information it gets-Deep Blue lacks all of these. I think the best functional account of human consciousness is given by the distinguished Canadian neuropsychologist, Merlin Donald. He calls it "The Governor of Mental Life" which functions as the meaning-maker and manager in science, culture, and religion. About this he wrote:

What consciousness is really about, at least in the human species ... is much deeper than the
sensory stream. It is about building and sustaining mental models of reality, constructing
meaning, and asserting autonomous intermediate-term control over one's thought process,
even without the extra clarity afforded by the explicit consensual system of language.
The engine of the symbolic mind, the one that ultimately generates language to serve its
own representational agenda, is much larger and more powerful than language, which is
after all its own (generally inadequate) invention. ${ }^{9}$

Meaning-making —otherwise called "meaning-constitution" or "intentional activity"-is the making of concepts, predications, judgments against an appropriate a priori background of lifeworld, context, and practices. They all involve dialogically the specific subjective embodiment of the speaker as well as an intended environmental context for the discourse. For scientific discourse, a dialogical community lives in the context of a theoretical language and a scientific laboratory. In this analysis, I follow the way of hermeneutical and phenomenological thinking according to Edmund Husserl ([1952] 1989, 1966, [1901-1913] 1970a,

\footnotetext{
${ }^{8}$ The terms "perception" and "observation" are used in this article as synomynous.

${ }^{9}$ Donald (2001), 75.
} 
[1954] 1970b), ${ }^{10}$ Martin Heidegger (1962, 1967, 1982, 1995, 1999, 2002a, b), Maurice Merleau-Ponty (1962), Kockelmans and Kisiel (1970), and others. ${ }^{11}$

\section{Hermeneutics as the Universal and Transcendental Process of Meaning-Making}

The universal and transcendental process of meaning-making is a circular or cyclic process that is often called the "hermeneutical circle/cycle.' ${ }^{12}$ Each circle-or cycle-follows a sequence of four phases-a. experiencing/observing, b. theorymaking, c. theory-testing, and d. deciding —each phase giving access to new insights; each cycle leading to a partially transformed beginning of a new cycle in which further development is made. Each cycle revises and improves the previous cycles of inquiry until the basic queries have been sufficiently explored dialogically.

To exemplify the process of the hermeneutical circle, I will tell the story of the distinguished psychologist James J. Gibson's discovery of the non-Euclidean geometry of human vision while training young pilots to fly during the war. So many of these young men killed themselves when landing their planes that he came to suspect that the problem was not an engineering problem, but a human one related to spatial vision. He suspected that 'natural' human vision systematically estimated vertical altitudes differently from the way they are estimated by scientific measurement. Thus he was led to the hypothesis that the visual space of humans had a different geometry from the Euclidean. Some time in the early seventies, I was invited by the MIT Psychology Department to speak on the occasion of the celebration of Gibson's 70th birthday. I spoke about the work I was doing on the curved Riemannian geometry of Van Gogh's paintings and I spoke of the experimental studies of von Helmholtz and others on the non-Euclidean geometries of visual space. ${ }^{13}$ Gibson was pleased with my talk and responded by telling his story about why so many student pilots killed themselves when trying to land their planes because human vision without instruments is not adequate for flying. He said that based on this experience, he formulated a rule-now universally mandatory for all pilots - that, when landing a plane, they must rely exclusively on technological guidance, such as on-board instruments, instructions from the airport tower-or

\footnotetext{
${ }^{10}$ For an excellent guide to Husserl, see Welton (2000).

${ }^{11}$ All of these are linked with the ancient Greek and scholastic tradition through Bernard Lonergan's reflection on the transcendental process of meaning-making, and the importance of what he calls, 'interiority' Lonergan ([1957] 1992, [1972] 1990); 'interiority' is the awareness of oneself as being an embodied consciousness and as such, the Governor of one's Mental Life.

${ }^{12}$ See Kisiel (1970b); also Heidegger (1962). The scholastic tradition is a bridge that connects the classical tradition and phenomenology; for this reason, I find Bernard Lonergan helpful; see Lonergan ([1957] 1992).

${ }^{13}$ See Heelan (1983/1987).
} 
lacking these - they must follow the now standard "Gibsonian" markings on the ground approaching the airport. ${ }^{14}$

The four phases of the hermeneutical circle can easily be discerned when applied to Gibson's story: the experience of pilots' failures ${ }^{15}$; the theory/hypothesis of non-Euclidean vision; the theory-testing in experimental studies of binocular visual geometry ${ }^{16}$; and the decision to apply the consequences of binocular visual geometry to piloting planes. ${ }^{17}$

In his reflections on visual space, Gibson also asked himself about binocular vision in the context of human evolutionary history-whether a 'natural' binocular space, which is curved and of finite size, would have served early human communities in their 'natural' environment better than an infinite flat Euclidean space to which modern culture is accustomed. He concluded that 'natural' binocular curved visual space would be more useful, first because it highlights a nearby quasiEuclidean frontal zone for good eye-hand coordination, while more distant objects are projected without depth onto the visual dome, the one that rests on the horizon and rises to become the background for the clouds during the day and the stars during the night. From the point of view of cognitive science, however, the account of pure vision given above seems to be consistent with the dual visual neurological pathways that neuroscientists have found. ${ }^{18}$ For our early ancestors, however, and for ourselves today - should we strip away what science teaches us - the 'natural' meaning of pure vision is neither Galilean nor Einsteinian, but what comes from Grimm's fairy tales. ${ }^{19}$

Perhaps of even greater critical importance is the hermeneutical criticism of classical scientific research on human vision — such as Galileo's-in overlooking the dual role played by light_-for light is a physical medium subject to electromagnetic laws and it also carries a visual message about the environment. This dual function is often overlooked and-in the familiar words of Marshal McLuhan- "the medium is the message." The objects of visual experienceswhat we see-are not just the photons/rays of light falling on the retina but the

\footnotetext{
${ }^{14}$ See Gibson (1979).

${ }^{15}$ Ibid.

${ }^{16}$ See Heelan (1983/1988), passim, and the Appendix in which the history of the geometry of curved visual spaces is presented.

${ }^{17}$ Gibson found the hypothesis was reasonable in the light of biological evolution; that many everyday phenomena seemed to support it, and that the laboratory scientific made by H. von Helmholtz (c. 1876) and others such as R. Luneburg, A. Blank, T. Indow, J. M. Foley and others provide positive evidence.

${ }^{18}$ Jacob and Jeannerod (2003), Jacob (1988), Pribram (1991).

${ }^{19}$ The Visual Space of our early human ancestors was constituted by a nearby virtually Euclidean zone that Arnheim (1974) called the 'Newtonian Oasis,' and a far zone that surrounds it where the depth of field diminishes rapidly to zero Heelan (1972, 1983, [1983] 1988), Part I and Appendix; Luneburg $(1947,1985)$. In theory, the non-Euclidean geometry of natural human visual space can be derived a priori from stereoscopy. The characteristics of this general structure have been confirmed by testing (Luneburg 1947, 1895 ; Heelan 1972, 1983, [1983] 1988).
} 
information they carry about the environment; what the photons/rays show is not themselves but the presence of distant three-dimensional environmental bodies which are their source. As human visual organs receive the incoming stream of photonic messengers, they draw from them environmental information appropriate for action. Among such action are the coordination of hands, eyes, limb movements, and possibly instrumental controls. ${ }^{20}$ As physical entities the photon-messengers move in Galilean/Euclidean physical space, but they invite interpretation by human embodied consciousness, who consequently sees an illuminated space of physical objects in the curved visual space inherited from our biological ancestors. In this curved space, there is a local privileged zone where hand-eye coordination is quasi-Euclidean. Distant objects, however, are given only in superficial profiles on the surrounding celestial dome. The geometric family of such visual spaces, as I have said, can be inferred a priori from the theoretical treatment of binocular stereoscopic vision. The curvature of such visual spaces plays an active-and often disconcerting and dangerous roleparticularly, in engineered environments, such as modern highways, and-as Gibson found-in guiding planes to safe airport landings. The conclusion that Gibson came to was, that 'natural' human vision was shaped for terrestrial living and not for living in the air like birds.

The hermeneutical circle, as I have said, is the structure of the transcendental rationality of dynamic human consciousness. ${ }^{21}$ This is not simply what is usually understood as Enlightenment Reason or objective science. Self-awareness of this transcendental dynamic embodied function constitutes a rare virtue that Bernard Lonergan calls 'interiority' ${ }^{22}$ which is discernable in the writings of ancient and modern authors, from Plato and Aristotle to Aquinas, and up to the present time. Heraclitus once said that human consciousness loves to hide itself - a sentiment shared with many psychologists, cognitive scientists, social scientists, physicists, and philosophers. ${ }^{23}$ Such a sense of the embodied-self-in-the-world is reflected in a special way in the phenomenological writings of Husserl, particularly in his later works, also in Merleau-Ponty's Phenomenology of Perception (1962), and in Heidegger's Being and Time (1927/1962).

'Interiority' ts a virtue of human consciousness that is also exemplified in the views of at least the four physicists I mentioned at the head of this article, namely, Schrödinger, Wigner, Dirac, and Heisenberg. 'Interiority' makes deep demands on philosophers and cognitive scientists, especially on those concerned with the rationality of contemporary physics, cognitive science, ethics, and religious faith.

\footnotetext{
${ }^{20}$ See Berthoz and Petit (2008).

${ }^{21}$ See Heelan $(1994,1998)$.

${ }^{22}$ See Lonergan (1957/1992).

${ }^{23}$ See Hadot (2006), Chap. 1.
} 


\section{The Governor of Mental Life and Meaning-Making}

The Governor of Mental Life-human consciousness - makes meanings of different kinds. We turn next to meaning-making in the natural sciences. Many different kinds of meanings are made in the natural sciences, such as concept and category formation, theory formation, theory testing in the laboratory, and theory affirming.

About concept/category ${ }^{24}$ formation: We ask first: What are 'concepts' ontologically? How are they constituted? Are they 'local/contextual' invariants/likenesses/ symmetries $^{25}$ of an a posteriori set of particular empirical instances/events held in the memory as alike in some categorical way and likely to be changeable over time? Or are they a priori 'unchangeable/transcendental' ideals, expressed, say, in mathematics or pure logic, with respect to which any empirical instance/event absolutely and necessarily conforms?

Whatever concepts are, and however constituted, they are represented by mathematical and linguistic media of communication: It is then necessary to distinguish the two uses of the representing medium: the medium as messenger, and the information carried by the messenger for delivery to appropriate interpreters-speakers and hearers. The nub of Heisenberg's and Einstein's problem referred to above was how to distinguish and relate the medium and the message in order to make sense of the quantum Uncertainty Principles.

About theory formation: In particular, what are the distinguishing linguistic roles of mathematics and logic in the formation and use of theory? In terms of "grammar" and "lexicon," the 'lexicon' of a science refers to what is 'observable' in the process of measurement, and the 'grammar' of the science refers to its mathematical theoretical structure where 'intuition' has its place.

About theory testing: Theory testing leaves a residue of meaning uncertainty due to the contingency of empirical evidence. Contingency is a function of the variety of possible contextual ${ }^{26}$ circumstances implicitly intended in the instantiation of an 'observable,' such as the evidentiary horizon of the laboratory, the social demand for cultural and institutional agreement, the historical dimension of languages, practices, cultures, institutions, etc. These implicate social, cultural, and historical aspects of natural science, as well as, say, the philosophical and theological culture of the local environment. They demand of the speakers/hearers an attitude of continual prudent review and revision. The natural sciences are evidently not finished products. Ethical, aesthetic, and religious meaning-making, as well as other value-added aspects of decision-making, serve to condition the choices of the inquirer as well as the chosen circumstances of the inquiry.

\footnotetext{
${ }^{24}$ For the purposes of this paper, I do not distinguish between "concept" and "category."

${ }^{25}$ I use the terms "invariant," "likeness," and "symmetry" interchangeably; they define the same group-theoretical quality which remains constant despite merely perspectival changesrepresented usually by group-theoretic transformation laws of space and time.

${ }^{26}$ See Heelan $(1974,2003)$ and Hasan (2010).
} 
The complex canvas sketched out above is large, but I intend to cover just as much as is necessary to show how phenomenological and hermeneutical considerations force one to move beyond current science-speak to find the observable (ontological) reality that science noetically and epistemologically intends. ${ }^{27}$

Evolutionary Concept/Category Formation: Descriptive category formation is part of the general story of human evolution! What is it about category-making that makes the human sciences hide the emergence of human consciousness in the story of evolution? Human infants do not enter the world conscious of knowing anything about it, but they enter equipped with all that is necessary to learn from their environment. They learn from adults around them by 'reading their minds,' communicating by 'mimicry,' and later by 'language,' exploring their environment for observable content, and eventually expressing what they mean in the language of the family or caregivers. Finally, they learn to collaborate with their family and caregivers who by their natural authority introduce them to their local world, and to the means to share it, and to represent it through language as members of a human community. ${ }^{28}$

Concept/Category Formation of Observable Objects: The process of observation (perceptual recognition) supposes a descriptive category that is associated with a lexical name, an observational praxis, and a standard sensory medium of representation. How is the category that goes with that lexical name constructed? A Husserlean phenomenological analysis ${ }^{29}$ would describe its intentional constitution as the creation or recognition of a symmetry (an invariant and repeatable pattern or likeness) present in a set of individuals 'given' to observation amid the flux of sensations by the human learned art of interpreting visual stimuli. ${ }^{30}$ Learning of this kind is an interpretative/hermeneutical process structured both by nature and by culture. ${ }^{31}$ It is more primitive than, say, the reading of a text, since the reading of semiotic textual signs already presupposes an acquired cultural resource from which to draw. One function, then, of the Governor of Mental Life is to reveal an intuited meaningful symmetry that is 'given' in observation because 'found' in a flux of local embodied sensation within a local enframing practical context of observer and observed. The mathematical structure in this account supposes an intuited group-theoretic symmetry, made present by a learned praxis of observing, by rendering meaningful by 'interpretation,' the sensory flux. Something — let us call it a "symmetry" —is found

\footnotetext{
${ }^{27}$ Kisiel and Kockelmans address these philosophical questions from within the language of Husserl and Heidegger; I approach them here from the scientific side, showing how scientists have failed to reach out hermeneutically beyond their models and their "data" in order to re-discover what is ontologically present but hidden in the measured "datum"; ref. Kisiel and Kockelmans (1970), especially Kisiel (1970c) and Kockelmans (1970b).

${ }^{28}$ See Tomasello (1999).

${ }^{29}$ See Jacob and Jeannerod (2003), Jacob (1988), Pribram (1991).

${ }^{30}$ Husserl makes an important distinction between (1). 'experience' which is intentional in relation to ontological reality and the core of the pure phenomenology of experience, and (2). 'experience' which is 'inner consciousness/perception' and the content of the former, see Husserl (1970a), Investigation V, 542-545. See also Cassirer (1944).

${ }^{31}$ See Tomasello (1999).
} 
and defined by a common likeness among the set of canonical exemplars, chosen to be held in memory. Each canonical exemplar held in memory is related to the others then as (Husserlian) 'profiles' of the same symmetry. The members of the canonical exemplifying set are updated periodically, with new exemplars replacing old ones, leading thereby to a shift in the meaning of the symmetry. The category is then defined by the symmetry exemplified by an appropriate set of canonical particulars ('profiles'). The category represents an invariant that involves the observer, a canonical set of observed exemplars in memory, and a standard enframing of physical and cultural context.

However, canonical exemplars which exemplify a particular symmetry, say, being a 'ball,' can nevertheless fail to exemplify other symmetries, such as "roundness"- for a 'football' (in the USA and in the case of rugby in the UK) is not round, though it is round in the rest of the world. The category of 'ball' then has an uncertainty relation to 'roundness.'

Concept/Category Formation by Measurement: Measurement gives a 'numbered datum.' Returning to the Heisenberg/Einstein problem referred to above, a numbered datum in quantum physics could be no more than a present messenger. The message it carries, however, has to be 'read' from the messenger-taken-as-code, the messenger as 'information.' To get the message from the information, the coded message has to be 'interpreted.' In the case of a measurement, the message is the datum; the datum is real, and present in the laboratory (together with the other theoretical observables functionally related to it). However, nothing more is communicated by the messenger-as-code than the ontological presence in the laboratory of the 'observable' —now as the 'observed.' But while 'observation' is generally accompanied by the intuition of place, shape, size, color, etc. these common lifeworld qualities are absent and seemingly irrelevant. An act of quantum measurement then is an 'observational' act, performed by an 'observer' conscious of being embodied in the laboratory, but blind to the common sensual intuitions of the lifeworld; in the paradigmatic way, the quantum observer 'embodied' in the laboratory has the "consciousness of a blind man" 'embodied' in his cane, inhabiting it with his bodily sensibility, and capable, for instance, of intuiting his local lifeworld space, but incapable of intuiting its colors. ${ }^{32}$

\section{The Role of Theory and Laboratory Context in Meaning-Making}

Pure Mathematics, Anschaulichkeit/intuition, Meaning Uncertainty: Puremathematics is the pure science of meaningful structure, it is a set of defined formal relationships among a lexicon of postulated mathematical entities — whether numbers, figures, or patterns - that inhabit the space of the mathematical (algebraic or geometrical)

\footnotetext{
${ }^{32}$ See Merleau-Ponty (1962), Heelan ([1983] 1988).
} 
imagination. These mathematical entities exist only as intuited-in German, as anschaulich - in the esthetic space of the mathematical logic or imagination.

Theoretical Physics and Mathematical Models in Physics: The essence of modern science is historically the mathematizing of the measured world. Mathematical points, lines, and surfaces, however, are not empirical bodies in the world; they are pure-non-empirical-elements defined within mathematical intuition by algebraic or geometric functions. In relation to the real sensible world, an intuited representation in the imagination is no more than a semiotic element like a lexical word of text or like a syntactical structure of grammar. ${ }^{33}$ It can be used, however, in a predication of experience the way a pure concept is used; such a predication instantiates the mathematical representation of a physical exemplar.

How is this done? A theoretical computation is a function in the field of mathematics. The classical mathematical field is the field of Anschaulichkeit, the field of logical structures and functions intuitable in Space and Time as imagined. ${ }^{34}$ Mathematical formulas can be used to symbolize operations in the 'real' sensible world, usually through the instrumentality of measurement, thereby associating a network of measure-numbers with a network of related and named physical properties. Through such a mathematical model, real aspects of the empirical spatio-temporal world of human culture can be ordered and controlled. ${ }^{35}$

As a function related to human evolution, mathematical intuition is a cultural development of the primordial human ability to see, hear, touch, taste, and feel the world perceptually by recognizing recurrent patterns in the sensory flux, such as the numbers-measure-numbers - supplied by a laboratory measurement. These real patterns of measure-numbers, accessible by measurement, can then be used to represent an ontological entity-sometimes misleadingly called a 'theoretical entity.' The measure-number is not 'what is meant,' it is itself no more than a messenger that is a symbol of the empirical presence of something real in the space-time of the laboratory but which possibly is not imaginable or intuitable. Observation in this way is organized mathematically by measurement as a medium to explore 'what is' but what may not be imaginable/intuitable in any of the spacetimes with which we are familiar. Mathematics as coding introduces the essential evolutionary function of mathematics which has its own esthetic and practical value while it also has the capacity to point beyond itself to something ontological. Though mathematics has a transcendent esthetic beauty for professional mathematicians, it is not a divine language, as some distinguished physicists have piously speculated. It certainly is a human language as its history shows; it is one, however, that serves rather the function of a 'grammar' than that of a 'lexicon,' and is closely connected with the way we embodied humans organize our world by number codes, naming recurrent patterns among exemplars despite evident differences among them that produce uncertainties.

\footnotetext{
${ }^{33}$ See Hasan (2010). For a more phenomenological presentation, see Kockelmans (1970a) in Kockelmans and Kisiel (1970).

${ }^{34}$ For the hermeneutic foundations of mathematics, it is worth looking at Lakoff and Nunez (2000).

${ }^{35}$ See Ryckman (2005), Heelan (2003, 2004).
} 
Among the basic organizational skills we have is the native ability to find patterns in the sensory flux to which we assign a meaning that is public, and shared through language (or a language substitute) with our cultural community. ${ }^{36}$ Such shared and recurrent meanings are based on two kinds of recognized patterns in the sensory flux: anschaulich (intuitively meaningful) space-time patterns, and intensional, ${ }^{37}$ (categorically meaningful). The former is the symmetry (invariant) that characterizes abstract mathematical intuitions that are universally valid in principle for all mathematically oriented communities; the latter is the symmetry (invariant) that characterizes observation and measurement, both of which are contextualized by local empirical circumstances, communities, needs, and goals.

Classical physics is the natural science which has faith in assuming that the observational world is simply the instantiation of culture-free a priori ideal mathematical objects. It is clear, however, that in certain situations elementary particles have to be treated differently from geometric points in space and time. Quantum physics seems to have good theoretical and experimental reasons for giving up faith in the identity of physics with mathematics. ${ }^{38}$

This should not have been a surprising discovery in the context of human evolution, since there is little likelihood that human visual and tactile perception would have been shaped by any other practices than those that coordinate the local actions of eyes, ears, hands, and legs which privilege a range of what turns out to be nonEuclidean finite visual spaces. ${ }^{39}$ Cosmological matters of human interest, such as seasonal and weather changes, were treated by reading the signs in the heavens and in other ways; while matters of health and nourishment were managed by taste and smell, and by reading Nature's 'signatures' in plants and animals. There is then no a priori reason from evolutionary principles to justify universal scientific trust in the Anschaulichkeit criterion of the modern scientific human imagination. Such a trust was inherited mostly from the early modern period of European cultural history when the mathematization of the physical world — small, medium, and large — came to be incautiously accepted as fundamental. In recent times, it has gradually become evident that the very small and the very large need their own lexicon-linked possibly to a common overarching transcendental 'grammar.' An important contribution

\footnotetext{
${ }^{36}$ For the grammar of scientific discourse, see Rheinberger (1997), Berthoz and Petit (2008), and also below.

${ }^{37}$ The terms 'extension' and 'intension' belong to mathematics and classical logic; extension connotes quantitative meanings (numbered or spatio-temporal), intension connotes cognitional (conceptual, logical) meanings. However, contrast this with the term 'intention,' differing slightly in spelling, on which account it is regularly confused with 'intension.' 'Intention' connotes purpose or intent and is related to action and experience. A derivative term, 'intentionality,' is central to a kind of philosophy that deals with how the meanings we make involve human action and experience. This is the philosophical 'phenomenology' associated with Edmund Husserl, Maurice Merleau-Ponty, and Martin Heidegger.

${ }^{38}$ See Heelan $(1965,1974,1975,1979,1987,1988)$.

${ }^{39}$ See Heelan ([1983] 1988), Appendix.
} 
to this end was made by Wigner and others ${ }^{40}$ in introducing (what is now called) the 'standard' form of the quantum theory; this is a Hilbert (infinite-dimensional vector) representation of Space that functions like a 'grammar' in which both classical and quantum entities can be represented, the classical by universal symmetries, and the quantum by local contextual symmetries. ${ }^{41}$

Hilbert Vector Space as the Grammar of a Science: Operators on Hilbert Space vectors represent practical measurement procedures that link human consciousness observationally with the micro-systems represented by the vectors in a Hilbert Space. ${ }^{42}$ The 'grammar' of those micro-systems represents not 'what is,' but how 'what is' is structured and structurally related. The scientific world we live in is then constituted existentially of universal 'absolute' symmetries, such as free classical entities, and 'local contextual symmetries'; this is the micro-structure of the laboratory world. Quantum physics has discovered a strange new property of spin that reaches across all Space and Time to function as a global link among micro-systems in cosmic nature. This global linkage exemplifies one of the kinds of global "entanglement" 43 in the scientific world. Such properties, while they stretch the powers of scientific intuition and observation beyond their natural (instrument free) human limits, serve to supply the intelligible foundation for the difference between the stable objects familiar in the everyday world we live in, and the instability of its dynamic foundations. Classical Space and Time can be seen in this perspective as the invariant (or symmetry) of a stable, but historically changing, human environment, rather than an invariant of the pre-existing unstable foundational world, the existence of which humans have come to recognize only lately. Other spaces, such as the variety of cultural visual and musical spaces in the course of historical time, belong to the domain of local contextual spaces and times. Quantum entities-as we know them today-seem to belong contextually to the unstable dynamic foundation of stable local historical cultural worlds.

How then are we to understand and represent to ourselves the 'quantum micro-realities' that appear fleetingly in laboratory experiments, or the anomalous 'cosmological macro-realities' — such as 'dark matter' and 'dark energy' — that appear in astronomical studies of the cosmos? Each makes its ontological 'presence' known in the laboratory where its measure-numbers appear. According to Heisenberg and Einstein (see above), however, the measure-number is no more than a 'code for presence' of what Heisenberg called "the observable," and not a 'description' of what exists ontologically. The ontology then-if knowable-has to be expressed in a (more) fundamental grammatical/mathematical language_-(let us call it) "F-spacetime"- - which is 'beyond' and 'deeper than' the 'mathematical space-time' of laboratory measurement. 'What is' - namely, the observable - is not describable in the space-time occupied by the messenger-lexicon, but (presumably) in some currently

\footnotetext{
${ }^{40}$ See Wigner (1962, 1963, 1967), also Wheeler and Zurek (1983), Dirac (1930), von Neumann (1955).

${ }^{41}$ See Heelan (1974, 1979), Bracken (2003).

${ }^{42}$ See Wheeler and Zurek (1983), Heelan (2004).

${ }^{43}$ See Aczel (2001), Shimony (1997), Gernert (2005).
} 
unknown "F-space-time," which is the context of the lexicon's message; F-space-time would then be the (currently unknown) foundational 'grammar' for the lexicon's message-language.

In summary, in the present state of micro- and cosmo-physics, both affirm the ontological laboratory presence of fundamental physical systems, but fail to be able to describe them ontologically in any current intuitive version of laboratory space-times. Let us suppose that there is yet a more fundamental logical and epistemological space-time beyond that of any current laboratory-F-space-time-in which those entities can be described, and about which the measure-numbers 'speak.' Such a fundamental space-time would (presumably) continue to be characterized by (grammar-based) epistemological intuition regarding the context of that about which the measure-numbers 'speak.'

This conclusion agrees with the outcome reached by Heisenberg and Einstein in Berlin in 1926. They agreed that the laboratory measure-numbers indicate the ontological presence of a micro-system - the 'observable' - within a context of measurement that does not provide an ontological description of the 'observable' micro-system in terms of the space-time of the measuring laboratory. What does the latter part of this claim mean?

In a phenomenological analysis, it means first, that the laboratory with its measuring instruments belongs to 'the extended body' of the observer, and thus, that the observer is 'the embodied human consciousness so extended.' Consequently, the observer lives in and through the laboratory measuring instruments as oriented towards practice; this is the channel of his/her 'noetic' intentionality. Under such circumstances, the measure-number becomes just a coded messenger, and like the photons received by the eyes in seeing, the message delivered by the measurenumbers is interpreted within the context of the measurement. In vision, it is not the photons which are 'observed' by the culturally prepared viewer, but the illuminated objects; so also in measurement, the measure-numbers are not what are 'observed' by the culturally prepared 'observer' but what they point to beyond themselves, namely, the presence of micro-systems in the laboratory situation, unaccompanied, however, by any intuitive description of them in the space-time of the laboratory.

Clearly then the ontology of quantum micro-entities in current quantum physics is not defined by human intuition [Anschaulichkeit]. To the extent that mathematical theory is the formal structural criterion of the 'language of physics,' its function is closer to that of grammar in linguistics that structures the lexicon of the lifeworld antecedent to observing and describing events in the lifeworld. The lexicon, however, names the categories of the things, actions, and values which exist for a local dialogical community. The criterion of Anschaulichkeit then is not, in this historical phase of human scientific culture, the basis of universal natural laws.

The Uncertainty of Meaning-making: 'Thin'versus 'Thick' Descriptions. ${ }^{44}$

1. 'Thin' description: Laboratory science and other abstractive academic disciplines give thin descriptions. These are descriptions that are narrowly contextualized, and

${ }^{44}$ See Geertz (1973), Chap. 1, and Williams (1985), 129-152. 
theoretically (abstractly) defined. The life of a modern scientific community, shielded as it is from having to take account of the diversity of surrounding cultures, horizons, agenda, styles, and goals, etc. is permeated with thin descriptions

2. 'Thick' description: Thick descriptions are practical 'world-guided' descriptions of actions or events that take account of the local, historical, multi-contextual, and multicultural niches where dialogue takes place. Thick descriptions permeate practical common life. Cultural knowledge is thick because it involves inter-contextual discourse among speakers and hearers whose skill in such discourse is not narrowly disciplinary but culturally dialogical. Aristotle included it under the character of prudence, "phronesis." This kind of discourse requires respect for the complexity and diversity of issues and authorities that characterize human cultural activity. The structure of the communicative exchanges in this kind of discourse is more like that of quantum theory, that is, one based on the choice of some relevant localized contextualized symmetry shared by all parties to the discourse.

\section{Dialogical Syntheses and the Grammar of Hilbert Space}

'Thick' dialogical discourse is exemplified in the history of science, for example, in the work of Ludwik Fleck (1979) who discovered the nature and source of the venereal disease, syphilis. He narrates how this discovery came to him. It was by re-interpreting a selection of old 'facts' from dialogical sources as diverse as 'old wives tales' and odd pieces of popular medical lore, in addition to the outcomes of his experimental work. He found in his community's public memory many of the ingredients from which he retrieved the insights which led him to define the two new scientific facts that have made him famous: the disease now known as 'syphilis,' and the 'spirochete' that causes it.

Rational Dialogical Synthesis: Classical and Quantum Science. The initial conflict between classical and quantum physics is a version of the pre-Socratic question: If Nature hides behind classical physics, what of the Nature that hides behind quantum physics? Can they be the same Nature? The key terms in the dispute often seem to be 'objectivity' and 'subjectivity', but in fact the key notions relevant to the transition from classical to quantum physics are 'intuition' [Anschaulichkeit] in the mathematics of quantum physics and 'measurement' in data acquisition and management. These terms, basic for understanding Niels Bohr's notion of 'complementarity, ${ }^{45}$ are today clamoring for re-examination.

Wigner and Dirac proposed a resolution of the dialogical conflict: The grammar and lexicon of both kinds of physics could be represented by a Hilbert Vector Space. In such a Space, a quantum system in pure (unobserved) motion is represented as developing under the influence of the appropriate Schrödinger equation; observed

${ }^{45}$ See Beller (1999). 
data, however, are represented mathematically by the 'eigen' (definite proper) values of the data operators acting on the state vector representation; these eigen values are codes for observable states of the system.

A dialogical conflict arose about the ontological and epistemological criteria of 'truth' and 'reality' in physics; it came to focus on the question of how mathematical intuition can be reconciled with observations to produce a coherent human understanding of the 'natural' world. Mathematical intuition is the a priori working space of the theoretical physicist; it structures - as it were grammatically - the a priori of the quantum narrative. Measurement defines the a posteriori observational space of the experimental physicist by providing the codes-measure-numbers-which enable the narrative of the observed data to be told. By introducing 'complementarity,' Bohr fudged the answer by attributing reality — of a classical kind — to the pure (ideal) objects of mathematical theory, while placing limits on observational access to these classical realities by measurement. In Kantian (and Neokantian) terms, mathematics describes the noumenon, while 'complementarity' restricts the observed phenomenon.

Deeply involved in all of this is the function of mathematics in any pure (nonempirical) discourse about the real world. In classical physics, its traditional function was to provide the intuition-Anschaulichkeit—of a generalized universal pure Space and Time that comprehensively 'represented' the physical world. This intuition was the sole reality guarantee of the 'representation' provided by physical theory. Such a view of mathematics goes back to Plato's 'likely story' in the Timaeus. Two millennia later, we find ourselves telling a different story!

Currently, there is a breakdown in dialogical common sense, because the traditional classical (Platonist) connection between a universal anschaulich space-time and its atomic contents fails in both quantum and relativity physics. The breakthrough was made by Eugene Wigner, Paul Dirac, and John von Neumann. Wigner ${ }^{46}$ was the key figure in this proclamation. ${ }^{47} \mathrm{He}$ was trained as a physical chemist and crystallographer, familiar with the chemical laboratory. His mentor was Michael Polanyi $^{48}$ also a physical chemist and later a well-known philosopher of science. Wigner applied to quantum theory the kind of dynamic group theoretic mathematics that crystallographers use to describe the production of crystalline symmetries in a solution, and to explain mixed crystalline forms in ways that foreshadowed the uncertainties of quantum physics. Wigner represented the state of a quantum system by an infinite-dimensional vector in a Hilbert Space governed by a dynamic law of change and development inspired by Schrödinger.

Wigner was Hilbert's assistant at the University of Göttingen from 1927 till 1931. There he reflected deeply on the higher purpose of physics, it was "to elevate the material side of the world, to make daily life easier for all the world's people." 49

\footnotetext{
${ }^{46}$ See Szanton (1992), 309.

${ }^{47}$ Wigner was also the brother-in-law of Dirac, and a schoolboy chum of von Neumann in his native Hungary.

${ }^{48}$ See Scott and Moleski (2005).

${ }^{49}$ Szanton (1992), 111; see also 308-309.
} 
The academic environment at the university was steeped in Husserl's philosophy and the notion of "invariance/symmetry" that defined its core. Wigner introduced what is now the standard form of the quantum theory. In this representation, states of the quantum system are represented by an infinite-dimensional vector in mathematical Hilbert Space. In the new mathematical representation, observablesproperties of the system empirically accessible-are represented by projection operators on the Hilbert Space. A projection operator generates a subspace of the Hilbert Space in which the relevant observable has a definite ('eigen') value. Other observables applied to this subspace are permitted to have definite values provided their projection operators are compatible with it (by algebraic commutation), but not otherwise. What is new about the Hilbert Space representation is its capacity to represent and encode contextuality: pairs of observables which share a common context can be measured together, while pairs which do not share a common context, cannot be measured together with certainty. Relative then to quantum systems, observables are context-dependent; some pairs can be measured accurately together, other pairs cannot be measured accurately together and suffer uncertainty. The great achievement of Wigner and Dirac was to generate a mathematical representation of the context-dependency of observables in quantum mechanics. ${ }^{50}$

In the standard [Hilbert Vector Space] view of quantum physics, the 'properties' of physical objects are expressed as a combination of locally context-dependent 'observables' (represented by contextual 'invariants' or 'symmetries') within the universal symmetry group of space-time. Classical physics knows nothing about, and ignores local context-dependent properties. Hilbert Space quantum physics revolutionized the science of physics because, by allowing for different local and mutually incompatible contexts or horizons of empirical research, the physicist can unify her search by drawing on a broader heuristic question: whether the unknown $\mathrm{X}$ (that is sought) defines a universal symmetry of the Hilbert Space or a local context-dependent symmetry-representing the relevant subspace-of the Hilbert Space. If I am researching a local symmetry, then I must design the kind of laboratory bench that allows me to observe the context-dependent effects of the local symmetry. The real world model now unifies both universal and local symmetries in a common synthesis.

The synthesis takes note of the presence and role of the embodied consciousness of the local observer as it functions in the measurement process, and who by choice and agency enters into the definition of the local group theoretic symmetry. Quantum physics then has to recognize the dependence of observables on the contextdependent physical platform of the chosen laboratory bench, insofar as the laboratory bench is an extension of the embodied character of the consciousness of the scientific observer. In contrast with observations in classical physics, observations in quantum mechanics from different local physical platforms (represented by different subspaces of the Hilbert Space) do not simply add up to a coherent objective culture-free scientific account.

${ }^{50}$ For these insights, Dirac received the Nobel Prize in physics in 1933; Wigner, for his part, in 1963. 
One consequence is that in quantum physics, it is not possible in principle to give a comprehensive empirical description of a unified and objective 'world' for all observers. There is no more than a unified and comprehensive grammar of the pure world-for-any-observer-namely, the Hilbert Space with its systems of vectors and operators; but for any individual observer, the observables of the world constitute context-dependent branches peculiar to that observer and that observer's choice of what to measure. All such knowledge is partial, relative, scientific, but culturally perspectival.

Is this, you ask, the little we are left with when natural science promised so much more? We should not forget that the little we know of the quantum world is accompanied by the bonus of knowing something about ourselves-namely, awareness of the interiority of the work of human consciousness embodied in the process of scientific measurement, just as in every act of perception/observation. This reflection warns us that we are an evolutionary product of Nature and cannot assume that we can study Nature 'objectively' from beyond the horizon of Nature. We rule Nature from within Nature — not as monarchs of Nature-but as its gardeners.

\section{Is a Theory of Human Consciousness?}

Phenomenology is concerned with human consciousness. Human consciousness, the Governor of Mental Life, is the agent that produces and recognizes the categories for a strict theoretical science. Can human consciousness produce the category to define itself?

Human consciousness produces categories and recognizes instances of the various categories by becoming aware of recursive patterns of form and function in the sensory flux. If the only source of categorial knowledge is the embodied sensory flux, then the question becomes: Has human consciousness access to the kind of embodied sensory flux that reveals the category to which human consciousness belongs? A category is a 'symmetry' ('invariant' or 'property') among a core set of embodied exemplars; it is normative for all instances of its kind; a categorial symmetry is theoretical to the extent that elements of the set can substitute for one another as exemplars of the symmetry. Human consciousness is peculiar in that while we have no trouble in practice recognizing exemplars of human consciousness-we call them "persons"-we are nevertheless hesitant to say that they can substitute for one another under a 'category' of 'persons,' in the way we count instances of cups, saucers, birds, and beasts. Persons can substitute for one another in specific ways, as car buyers, as music lovers, as sports' fans, as Democrats, as Republicans, Yes! but ... as 'human consciousnesses'? No! because people do not share their personal identity. Personal identity is expressed existentially in the individual's living 'interiority' or 'being-in-the world.' If others are 'persons' like me, they will be fundamentally different knowers and doers, though sharing many cultural contexts. Isn't this then what it means to understand oneself as a person, and as an exemplar of human consciousness? But this is not a categorial understanding of human consciousness, for there are few if any universally true and absolute 
deductive inferences that can be drawn from the descriptions of individual persons. Phenomenology, as the study of human consciousness, leads to a theoretical study of the dynamic, normative structures of intentionality, meaning-making, and decision-making, but not to the existential choices and practices that individually shape the human consciousness that is a Governor of Mental Life.

\section{Recapitulation}

To summarize what has been said in this paper: This paper seeks to exemplify how hermeneutical phenomenology can analyze the implicit meaning and context of the natural sciences, in particular the epistemological and ontological problems of quantum physics. In this light quantum physics is shown to be fundamentally a return from the transcendental world of classical physics to the lifeworld of human experience, in which the contextuality of scientific discourse is exemplified in observation, and the human subject becomes the human embodied consciousness of the observer which mediates the epistemic engagement with the ontology of the observed datum. New insight is given on the deeper meaning of the quantum uncertainty principles when examined philosophically from the viewpoint of hermeneutical phenomenology. The new emphasis is on the distinction between embodied subjectivity and observable objectivity, from which flow the epistemology and ontology of quantum physics. The ontology of quantum physics is clearly not culture-free and history-free, but whether progress can be beyond the present social and historical inventions of human consciousness within Nature still remains to be seen.

In this essay I claim that a Hilbert Space, exemplified by quantum physics, represents an existential grammar of the physical properties of the quantum system that is capable of synthesizing hermeneutically both universal and existential context-dependent concepts/categories. Quantum theory is then a phenomenological and hermeneutical theory of the phenomena of human consciousness, as claimed by Schrödinger, Wigner, and Heisenberg — and possibly Einstein. It is a theory of how embodied human consciousness - which is the Governor of Mental Life—acts as a new norm, extending the notion of human rationality beyond the classical norms of rationality to include hermeneutic norms and so to show the fundamental dependence of science on the lifeworld, as Husserl claimed and Kockelmans so brilliantly defended.

\section{References}

Aczel, Amir. 2001. EN Tanglement: The Greatest Mystery of Physics. New York: Four Walls Eight Windows.

Arnheim, Rudolf. 1974. Art and Visual Perception. Berkeley: University of California Press.

Babich, Babette. 1997. Against postmodernism and the 'new' philosophy of science: Nietzsche's image of science in the light of art. In Issues and Images in the Philosophy of Science, ed. Dimitri Ginev and R.S. Cohen, 27-46. 
Babich, Babette (ed.). 2002. Hermeneutic Philosophy and the Philosophy of Science, Van Gogh's Eyes and God. Essays in Honor of Patrick A. Heelan, S.J. Dordrecht: Kluwer.

Beller, Mara. 1999. Quantum dialogue: The story of a revolution. Chicago: University of Chicago Press.

Berthoz, Alain, and Jean-Luc Petit. 2008. The Physiology and Phenomenology of Action. New York: Oxford University Press.

Bracken, Anthony J. 2003. Quantum mechanics as an approximation to classical mechanics in Hilbert space. Journal of Physics A: Mathematics and Theoretical 36 \#23(13 June): L329-L335.

Byrnes, Heidi. 2002. The dialogism of meaning: The discursive embeddedness of knowledge, the colloquy of being. In Hermeneutic Philosophy and the Philosophy of Science, Van Gogh's Eyes and God, ed. B.E. Babich, 411-422. Dordrecht: Kluwer.

Cassirer, Ernst. 1944. The concept of group and the theory of perception. Philosophy and Phenomenological Research V/1: 1-35.

Dirac, Paul A.M. 1930. The principles of quantum mechanics. Oxford: Clarendon Press.

Donald, Merlin. 2001. A Mind so Rare: The Evolution of Human Consciousness. New York: Norton.

Eger, Martin. 1999. Language and the double hermeneutic in natural science. In Hermeneutics and science, ed. M. Feher, O. Kiss, and L. Ropolyi, 265-289. Dordrecht: Kluwer.

Einstein, Albert. 1950. Out of My Later Years. New York: Philosophical Library.

Farre, George. 1998. Characteristics and implications for the philosophy of nature. Acta Polytechnica Scandinavica 91: 3f.

Fleck, Ludwik. 1979. Genesis and Development of a Scientific Fact. Trans. T. Trenn. Chicago: University of Chicago Press.

Geertz, Clifford. 1973. The Interpretation of Cultures. New York: Basic Books.

Gernert, Dieter. 2005. Conditions of entanglement. Frontier Perspectives 14: 8-13.

Gibson, James J. 1979. The Ecological Approach to Visual Perception. Boston: Houghton Miflin.

Ginev, Dimitri, and Roert Cohen (eds.). 1997. Issues and Images in the Philosophy of Science. Dordrecht: Kluwer.

Hadot, Pierre. 2006. The Veil of Isis. Cambridge, MA: Harvard University Press.

Hasan, Ruqaiya. 2010. Describing Language: Form and Function (Collected works of Ruqaiya Hasan). London: Equinox Publishing.

Heelan, Patrick. 1965. Quantum Mechanics and Objectivity: A Study of the Physical Philosophy of Werner Heisenberg. The Hague: Nijhoff.

Heelan, Patrick. 1972. Towards a new analysis of the pictorial space of Vincent van Gogh. Art Bulletin 54: 478-492.

Heelan, Patrick. 1974. Quantum logic and classical logic: Their respective roles. In Logical and Epistemological Studies in Contemporary Physics, Boston studies in the philosophy of science series, vol. 13, ed. Robert S. Cohen and Wartofsky Marx, 318-349. The Hague: Reidel.

Heelan, Patrick. 1975. Heisenberg and radical theoretic change. Zeitschrift Für Allgemeine Wissenschaftstheorie 6: 113-138.

Heelan, Patrick. 1979. Complementarity, context-dependence and quantum logic. In The LogicoAlgebraic Approach to Quantum Mechanics, University of Western Ontario series on the philosophy of science, ed. C. Hooker, 161-179. Dordrecht: Reidel.

Heelan, Patrick. 1983. Perception as a hermeneutical act. Review of Metaphysics 37: 61-75.

Heelan, Patrick. 1987. Husserl's later philosophy of natural science. Philosophy of Science 54: 368-390.

Heelan, Patrick. 1983/1988. Space-Perception and the Philosophy of Science. Berkeley: University of California Press.

Heelan, Patrick. 1988. Husserl, Hilbert and the critique of Galilean science. In Edmund Husserl and the Phenomenological Tradition, ed. Robert Sokolowski, 157-173. Washington, DC: The Catholic University of America Press.

Heelan, Patrick. 1994. Galileo, Luther, and the hermeneutics of natural science. In The Question of Hermeneutics: Festschrift for Joseph Kockelmans, ed. Timothy Stapleton, 363-375. Dordrecht: Kluwer. 
Heelan, Patrick. 1998. Scope of hermeneutics in the philosophy of natural science. Studies in the History and Philosophy of Science 29: 273-298.

Heelan, Patrick. 2002. Faith and reason in philosophical perspective. In La Responsibilité de la raison: Hommage à Jean Ladrière, ed. J.-F. Malherbe, 149-175. Leuven: Peeters.

Heelan, Patrick. 2003. Phenomenology and the philosophy of the natural sciences. In Phenomenology World-Wide, ed. A.T. Tymieniecka, 631-640. Dordrecht: Kluwer.

Heelan, Patrick. 2004. The phenomenological role of consciousness in measurement. Mind and Matter 2: 61-84.

Heelan, Patrick. 2009. The role of consciousness as meaning-maker in science, culture, and religion. Zygon 44: 467-486.

Hefner, Philip. (ed.) 2006. On topics related to the quantum theory, reality, consciousness, spirit, and mind. Zygon: Journal of Religion and Science 41: \#3

Heidegger, M. 1962. Being and Time. Trans. J. Macquarrie, and E. Robinson. London: SCM Press.

Heidegger, M. 1967. What is a Thing? Orig. title, Die Frage nach dem Ding [1954] (trans: W.B. Barton, Jr and V. Deutsch). Chicago: Regnery.

Heidegger, M. 1982. On the Way to Language. Trans. P.D. Hertz. New York: Harper and Row.

Heidegger, M. 1995. Ontology (Hermeneutics of Facticity). Trans. J. van Buren. Bloomington: Indiana University Press.

Heidegger, M. 1999. Contributions to Philosophy (From enowning). Orig. title, Beiträge zur Philosophie [1989] (trans: Parvis Emad, and Kenneth Maly). Bloomington: Indiana University Press.

Heidegger, M. 2002a. Off the Beaten Track. Orig. title: 1950. Holzwege (trans. J. Young, and K. Haynes). Cambridge: Cambridge University Press.

Heidegger, M. 2002b. Supplement: From the Earliest Essays to Being and Time and Beyond, ed. J. van Buren. Albany: Starte University of New York Press.

Heisenberg, Werner. 1950. Physical Principles of the Quantum Theory. Trans. C. Eckart and F. C. Hoyt. New York: Dover. Orig. title: Physikalische Prinzipien der Quantentheorie [1930].

Heisenberg, Werner. 1971. Physics and Beyond: Encounters and Conversations. New York: Harper and Row. Orig. title, Der Teil und das Ganz. [1970].

Husserl, Edmund. 1966. Analyses Concerning Passive and Active Synthesis (Analysen zur passiven synthesis). Trans. A. Steinbock. Dordrecht: Kluwer.

Husserl, Edmund. [1901-1913] 1970a. Logical Investigations. Trans. J. N. Findley. New York: Humanities Press.

Husserl, Edmund. [1954] 1970b. The Crisis of European Sciences and Transcendental Philosophy: An Introduction to Phenomenological Philosophy. Trans. D. Carr. Evanston: Northwestern University Press.

Husserl, Edmund. [1952] 1989. Ideas II. Trans. R. Rojcewicz, and A. Schuwer Eng. Dordrecht: Kluwer.

Jacob, Francois. 1988. The Statue Within: An Autobiography. Trans. Franklin, Philip. New York: Basic Books.

Jacob, Pierre, and Marc Jeannerod. 2003. Ways of Seeing: The Scope and Limits of Visual Cognition. Oxford: Oxford University Press.

Kisiel, Theodore. 1970a. Phenomenology as the science of science. In Phenomenology and the natural sciences, ed. J. Kockelmans and T. Kisiel, 5-44. Evanston: Northwestern.

Kisiel, Theodore. 1970b. Husserl on the history of science. In Phenomenology and the natural sciences, ed. J. Kockelmans and T. Kisiel, 68-92. Evanston: Northwestern.

Kisiel, Theodore. 1970c. Merleua-Ponty on philosophy and science. In Phenomenology and the natural sciences, ed. J. Kockelmans and T. Kisiel, 251-273. Evanston: Northwestern.

Kockelmans, Joseph. 1970a. The mathematization of nature in Husserl's last publication, Krisis. In Phenomenology and the natural sciences, ed. J. Kockelmans and T. Kisiel, 45-67. Evanston: Northwestern.

Kockelmans, Joseph. 1970b. Merleau-Ponty on space perception and space. In Phenomenology and the natural sciences, ed. J. Kockelmans and T. Kisiel, 274-311. Evanston: Northwestern.

Kockelmans, Joseph, and Theodore Kisiel. 1970. Phenomenology and the Natural Sciences. Evanston: Northwestern University Press. 
Lakoff, George, and Rafael Nuňez. 2000. Where Mathematics Comes from: How the Embodied mind Brings Mathematics into Being. New York: Basic Books.

Lonergan, Bernard. (1957) 1992. Insight: A Study of Human Understanding. Toronto: University of Toronto Press.

Lonergan, Bernard. [1972] 1990. Method in Theology. Toronto: University of Toronto Press.

Lonergan, Bernard. 1950. The metric of visual space. Journal of the Optical Society of America 40: 627-642.

Luneburg, Rudolf. 1947. Mathematical Analysis of Binocular Vision. Princeton: Princeton University Press.

Luneburg, Rudolf. 1895. The metric of visual space. Journal of the Optical Society of America 40: 627-642.

Merleau-Ponty, Maurice. 1962. The Phenomenology of Perception. New York: Routledge and Kegan Paul.

Penrose, Roger. 1994. Shadows of the Mind: A Search for the Missing Science of Consciousness. Oxford: Oxford University Press.

Pribram, Karl. 1971. Languages of the Brain: Experimental Paradoxes and Principles in Neuropsychology. Englewood Cliffs: Prentice-Hall, Inc.

Pribram, Karl. 1991. Brain and Perception: Holonomy and Structure in Figural Processing. Hillsdale: Erlbaum.

Rheinberger, H.-J. 1997. Toward a history of epistemic things. Stanford: Stanford University Press.

Ryckman, Thomas. 2005. The Reign of Relativity: Philosophy in Physics 1915-1925. Oxford: Oxford University Press.

Scott, William T., and Martin X. Moleski. 2005. Michael Polanyi: Scientist and Philosopher. Oxford: Oxford University Press.

Shimony, Abner. 1997. On mentality, quantum mechanics and the actualization of potentialities. In The Large, the Small and the Human Mind, ed. Penrose Roger, A. Shimony, N. Cartwright, and S. Hawking, 144-160. Cambridge, UK: Cambridge University Press.

Szanton, Andrew. 1992. Recollections of Eugene Wigner. New York: Plenum.

Tomasello, Michael. 1999. The Cultural Origins of Human Cognition. Cambridge, MA: Harvard University Press.

Von Neumann, John. 1955. Mathematical Foundations of Quantum Mechanics, ed. R.T. Beyer. Princeton: Princeton University Press. Chap.VI: 417-445.

Wellmon, Chad. 2006. Poesie as antropology: Schleiermacher, colonial history, and the ethic of ethnography. The German Quarterly 79: 423-442.

Welton, Donn. 2000. The Other Husserl: The Horizons of Transcendental Phenomenology. Bloomington: Indiana University Press.

Wheeler, John A., and Wojciech H. Zurek. 1983. Quantum Theory and Measurement. Princeton: Princeton University Press.

Wigner, Eugene. 1962. Remarks on the mind-body question. In The Scientist Speculates, ed. I.J. Good, 284-301. London: Heinemanm.

Wigner, Eugene. 1963. The problem of measurement. American Journal of Physics 31: 6.

Wigner, Eugene. 1967. Symmetries and Reflections: Scientific Essays of Eugene P. Wigner. Bloomington: Indiana University Press.

Williams, Bernard. 1985. Ethics and the Limits of Philosophy. Cambridge, MA: Harvard University Press. 